\title{
THE EFFECT OF EXERCISE ON THE VOLUME OF THE BLOOD
}

\author{
By NOLAN L. KALTREIDER AND GEORGE R. MENEELY \\ (From the Department of Medicine, School of Medicine and Dentistry, University of Rochester, \\ and the Medical Clinics of the Strong Memorial and Rochester Municipal Hospitals, Rochester)
}

(Received for publication April 8, 1940)

The purpose of this investigation is to compare measurements of the blood volume and related factors in normal persons and in cases of heart disease before and after exercise. Previous indirect studies of the blood volume have indicated that as a result of exercise there is an increase in the concentration of the red blood cells and hemoglobin. Two explanations have been proposed to account for this change; $(a)$ the red cells are extruded from the blood depots into the circulation, thereby increasing the volume of the blood and cells $(1,2,3)$ and $(b)$ there is a change in the concentration of the blood due to passage of fluid from the blood into the tissues $(4,5)$.

The point at issue here should be readily clarified by direct measurements of the blood volume during and after exercise, but the results of such determinations do not settle the problem because there is no unanimity of opinion. In dogs after short periods of exercise one group of observers (6) found a decrease in the plasma and cell volumes, while another observer (7) noted an increase in all the components of the blood volume. In man, Chang and Harrop (8), employing the carbon monoxide method for the blood volume, exercised 4 normal individuals on a bicycle ergometer for from 6 to 10 minutes and found that the total circulating blood volume was increased from 1.3 to 7.3 per cent. Ewig and Hinsberg (9) and Wollheim (10) using the dye method found an average increase of 11.8 per cent in the plasma volume and 15.1 per cent in the cell volume after short periods of moderate exertion in normal subjects. Levin (11) determined the plasma volume immediately after exercise in 11 subjects and found it decreased in 8 subjects, unchanged in 1 and increased in 2 , while the cell volume was diminished in 4 , increased in 4 and unchanged in 3 cases. Lozoya (12), working at an altitude of 2249 meters, concluded from his observations that one-half hour of exercise (running and jumping) diminished the plasma volume 8.9 per cent, increased the cell volume 5.6 per cent, and decreased the total blood volume 4.1 per cent. Gibson and Branch (13) stated that they observed as a result of exercise a prompt and considerable decrease in the blood volume.

Observations on the blood volume before and after exercise in patients with heart disease are relatively few. Ewig and Hinsberg (9) and Wollheim (10) reported that, while in healthy individuals exercise increased the circulating blood volume through mobilization from the blood depots, in heart disease physical activity was not followed by such an effect, the volume remaining essentially the same. As the patient with heart disease regained his cardiac reserve, the response of the blood volume to exercise was again normal, i.e., an increase in plasma and cell volumes. The results of Levin (11) on heart disease are somewhat at variance with those reported by the above observers. This investigator found that the circulating blood volume increased in 58 per cent of the patients with heart disease (compensated and decompensated) in contrast to normal individuals in whom the volume was diminished in 73 per cent of cases as a result of exercise.

\section{METHODS AND PROCEDURES}

The plasma volume was measured by the dye method described by Gibson and Evans (14). The plasma volume is measured by determining the dilution in the blood stream of a measured amount of an azo dye, T-1824, after intravenous injection. Changes occurring during the experimental procedures were followed by the short "indirect" method (14). By this method changes in the plasma volume in excess of $+40 \mathrm{cc}$. and $-90 \mathrm{cc}$. may be considered significant. 1 The total blood and cell volumes

1 The assumption is made that the dye disappears from the blood stream at a linear rate and that the best line fitting the five initial points gives the rate. Actually the rate of disappearance of most substances from the blood stream is proportional to the amount present, i.e., it is an exponential curve. In this series of experiments, we were interested to know what was the magnitude of the errors which occurred, due both to the experimental variation and to the assumption that the disappearance is a straight line. In 2 cases observations on the concentra- 
were calculated from the plasma volume and the hematocrit values. The hemoglobin was determined by the oxygen combining power method (15), the blood viscosity by the Hess Viscometer and the proteins by Howe's Kjeldahl method (16). The circulation time was measured by the intravenous injection of "decholin" (17) and the venous pressure by the direct method of Griffith, Chamberlain and Kitchell (18).

The procedure of the experiments was as follows: All the subjects were examined under basal conditions. On coming to the laboratory in the morning the subject

tion of the dye in the serum were made for 90 minutes after the beginning of the experiment without doing anything to modify the blood volume. (See control curve, C. D., at the top of Figure 1.) The mean observed per cent error was -0.82 with a standard deviation of \pm 1.04 per cent. This means that, as far as one may argue from these 2 cases, 94 per cent of the observations will be between +1.26 and -2.90 per cent or $+38 \mathrm{cc}$. and -87 cc. for an average plasma volume of $3000 \mathrm{cc}$. rested on the seat of a bicycle ergometer for 45 minutes. During and for 10 minutes prior to the determination of the plasma volume the subject's arm was placed in a comfortable stand so adjusted that the antecubital vein was at the height of the angle of Louis. A vien in the antecubital fossa was punctured and blood was withdrawn for a dye free sample of serum. An accurately measured amount of dye was then injected. After rinsing the syringe and needle several times with blood the venous pressure was measured. Fifteen minutes (18 to $20 \mathrm{~min}$ utes in patients with heart disease) following the dye injection blood samples were taken at 5-minute intervals for 20 minutes. After the last sample was obtained the circulation time was determined.

When the sampling was completed, the subject was asked to ride a stationary bicycle for 10 minutes, the normal subjects performing on an average $400 \mathrm{kgm}$. per minute and the patients with heart disease $350 \mathrm{kgm}$. per minute. Samples of blood were withdrawn from the antecubital vein about half way through the exercise period and just before the exercise was terminated. Dur-

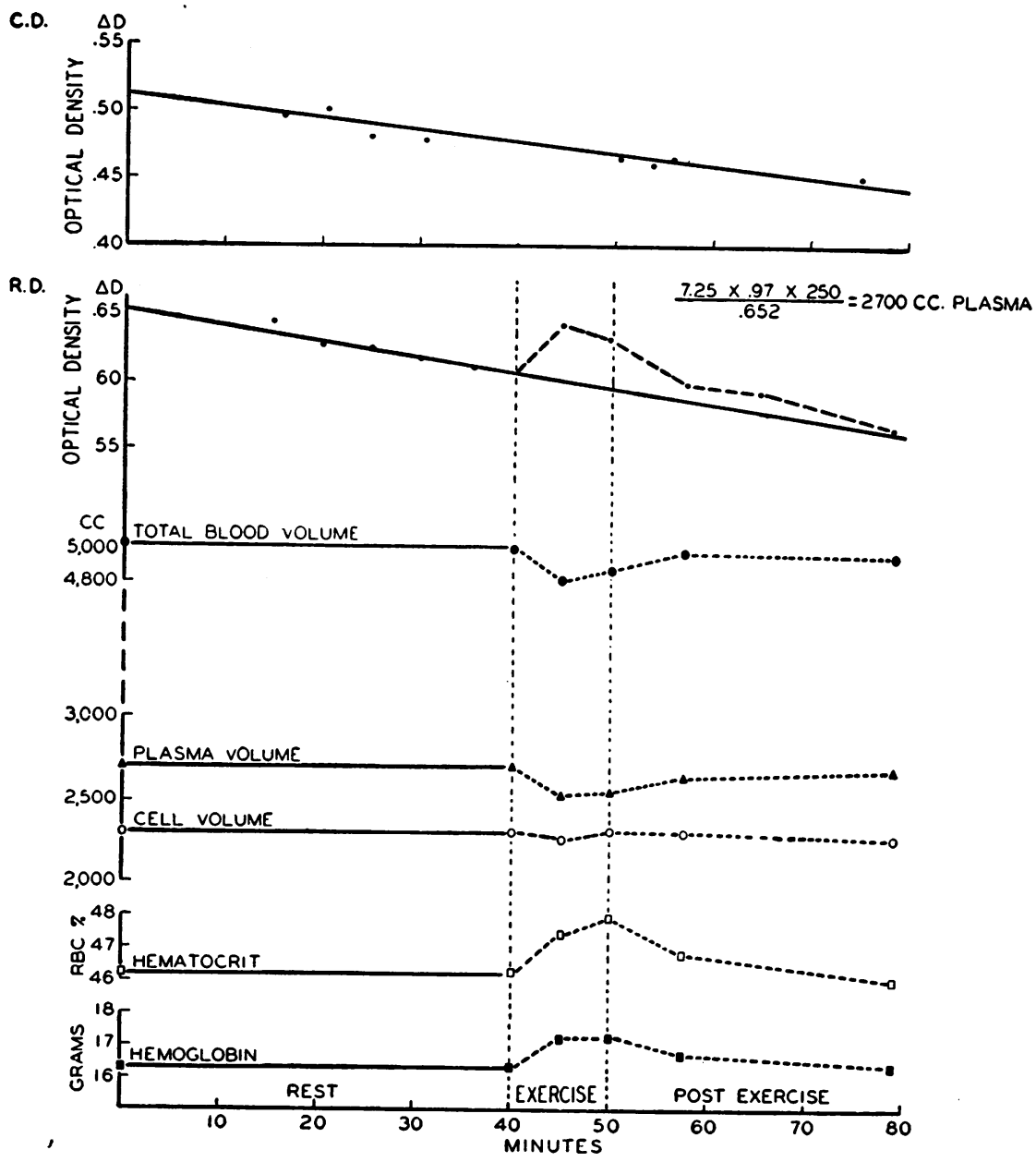

Fig. 1. The Alterations in the Volume of the Blood During and After Moderate Exertion in a Normal Individual 
ing the post exercise period blood was withdrawn for serum samples and relative cell volume. Circulation time and venous pressure were measured at varying intervals. The length of the experimental period was from 35 to 40 minutes.

In several experiments the type and amount of the work were changed somewhat. In one experiment (N. K.) the amount of work was doubled for 4 minutes after the usual period. In another, the subject (V. D.) rode the bicycle to exhaustion. The work in two other experiments (J. C. and A. R.) consisted of going up and down steps. In still another (A. M. B.) the subject was lying in bed while the blood volume was determined, after which he raised his legs above the bed and worked them vigorously for 10 minutes.

Determinations of the plasma and blood volumes were made before and following exercise on 6 normal individuals, 1 with chronic alcoholism, 1 with anorexia nervosa and 4 with compensated heart disease. In the latter group 3 of the patients had rheumatic heart disease; 1 had only involvement of the mitral valve, while the others had both aortic and mitral valves involved. The fourth patient of this group had hypertensive heart disease. All the patients had had one or more episodes of congestive heart failure. At the time of the determinations, all of the patients were ambulatory; all complained of dyspnea on moderate exertion but only 1 had signs of congestive failure, a slight peripheral edema. The cardiac reserve of these patients was not great, for all of them complained of rather severe respiratory distress on performing what is considered to be moderate exertion on the bicycle ergometer.

\section{RESULTS}

Normal subjects. The characteristic changes in the plasma and blood volumes during and after moderate exercise are depicted in Figure 1 and Table I. In every case exercise was accompanied by a prompt and definite decrease in the plasma volume. The diminution in the plasma volume was associated with an elevation in arterial and venous pressures. However, there was no correlation between the degree of increase in pressure and the decrease in volume. In 1 case,

TABLE I

Alterations in the volume of the blood before, during, and after muscular activity (normal subjects)

\begin{tabular}{|c|c|c|c|c|c|c|c|c|c|c|c|c|c|c|}
\hline & Conditions & \multicolumn{6}{|c|}{ Change in volume } & $\begin{array}{l}\text { Hema- } \\
\text { tocrit }\end{array}$ & $\begin{array}{l}\text { Hemo- } \\
\text { globin }\end{array}$ & $\begin{array}{c}\text { Serum } \\
\text { pro- } \\
\text { teins }\end{array}$ & $\begin{array}{l}\text { Total } \\
\text { circu- } \\
\text { lating } \\
\text { pro- } \\
\text { teins }\end{array}$ & $\begin{array}{l}\text { Vis- } \\
\text { cos- } \\
\text { ity }\end{array}$ & $\begin{array}{c}\text { Ve- } \\
\text { nous } \\
\text { pres } \\
\text { sure }\end{array}$ & Remarks \\
\hline $\begin{array}{l}\text { A. M. B. } \\
\text { January 11, } 1938\end{array}$ & $\begin{array}{l}\text { Rest } \\
\text { Exercise, } 6 \text { minutes } \\
\text { Exercise, } 10 \text { minutes } \\
\text { Post Exercise. } 19 \text { minutes }\end{array}$ & \begin{tabular}{c|}
$c$. \\
3600 \\
-480 \\
-430 \\
+40
\end{tabular} & $\begin{array}{c}\text { per } \\
\text { cent } \\
\\
-13.3 \\
-12.0 \\
+1.1\end{array}$ & $\begin{array}{c}c c . \\
6460 \\
-660 \\
-620 \\
+80\end{array}$ & $\begin{array}{c}\text { per } \\
\text { cent } \\
-9.9 \\
-9.3 \\
+1.6\end{array}$ & \begin{tabular}{r|}
$c c$ \\
2860 \\
-180 \\
-190 \\
+40
\end{tabular} & $\begin{array}{c}\text { per } \\
\text { cent } \\
\\
-6.3 \\
-6.6 \\
+1.4\end{array}$ & $\begin{array}{l}\text { per } \\
\text { cent } \\
44.3 \\
46.2 \\
45.7 \\
44.3\end{array}$ & \begin{tabular}{r|} 
grams \\
15.5 \\
16.1 \\
15.4
\end{tabular} & $\left|\begin{array}{c}\text { grams } \\
\text { per } \\
\text { cent }\end{array}\right|$ & grams & $\begin{array}{l}4.0 \\
4.4 \\
4.4 \\
4.3\end{array}$ & $\begin{array}{r}\operatorname{mm}_{2} \mathrm{O} \\
77 \\
282 \\
39\end{array}$ & $\begin{array}{l}\text { Sitting, bicycle ergometer; } 447 \mathrm{kgm} \text {. } \\
\text { per minute for } 10 \text { minutes. } \\
\text { Normal. }\end{array}$ \\
\hline$\underset{\text { March 29, } 1938}{\text { A. M. B. }}$ & $\begin{array}{l}\text { Rest } \\
\text { Exercise, } 6 \text { minutes } \\
\text { Post Exercise, } 2 \text { minutes } \\
\text { Post Exercise, } 8 \text { minutes } \\
\text { Post Exercise, } 28 \text { minutes }\end{array}$ & $\begin{array}{r}3600 \\
-960 \\
-560 \\
-250 \\
+30\end{array}$ & $\begin{array}{r}-26.7 \\
-15.6 \\
-7.0 \\
+0.8\end{array}$ & $\begin{array}{r}6240 \\
-660 \\
+100\end{array}$ & $\begin{array}{r}-10.6 \\
+1.6\end{array}$ & $\begin{array}{r}2640 \\
-100 \\
+70\end{array}$ & $\begin{array}{r}-3.8 \\
+2.7\end{array}$ & $\begin{array}{l}42.3 \\
45.5 \\
42.7\end{array}$ & $\begin{array}{l}14.6 \\
15.6 \\
14.8\end{array}$ & $\begin{array}{l}5.96 \\
6.68 \\
6.02\end{array}$ & $\begin{array}{l}215 \\
210 \\
219\end{array}$ & $\begin{array}{l}4.1 \\
4.6 \\
4.2\end{array}$ & & $\begin{array}{l}\text { Lying in bed, working legs vigor- } \\
\text { ously for } 10 \text { minutes, fatigued. } \\
\text { Normal. }\end{array}$ \\
\hline $\begin{array}{c}\text { R. D. } \\
\text { January 21, } 1938\end{array}$ & $\begin{array}{l}\text { Rest } \\
\text { Exercise, } 5 \text { minutes } \\
\text { Exercise, } 10 \text { minutes } \\
\text { Post Exercise, } 7 \frac{1}{2} \text { minutes } \\
\text { Post Exercise, } 25 \text { minutes }\end{array}$ & $\begin{array}{r}2700 \\
-170 \\
-150 \\
-50 \\
-20\end{array}$ & $\begin{array}{l}-6.3 \\
-5.5 \\
-1.8 \\
-0.7\end{array}$ & $\begin{array}{r}5020 \\
-210 \\
-140 \\
-40 \\
-60\end{array}$ & $\begin{array}{l}-4.2 \\
-2.8 \\
-0.8 \\
-1.2\end{array}$ & $\begin{array}{l}2320 \\
-40 \\
+10 \\
+10 \\
-40\end{array}$ & $\begin{array}{l}-1.7 \\
+0.4 \\
+0.4 \\
-1.7\end{array}$ & $\begin{array}{l}46.2 \\
47.4 \\
47.9 \\
46.8 \\
46.0\end{array}$ & $\begin{array}{l}16.3 \\
17.2 \\
17.2 \\
16.7 \\
16.3\end{array}$ & & & $\begin{array}{l}4.2 \\
4.5 \\
4.5 \\
4.3 \\
4.3\end{array}$ & $\begin{array}{r}77 \\
114 \\
84 \\
92\end{array}$ & $\begin{array}{l}\text { Sitting, bicycle ergometer; } 414 \mathrm{kgm} \text {. } \\
\text { per minute for } 10 \text { minutes. } \\
\text { Normal. }\end{array}$ \\
\hline $\begin{array}{c}\text { C. M. } \\
\text { March 2, } 1938\end{array}$ & $\begin{array}{l}\text { Rest } \\
\text { Exereise, } 5 \text { minutes } \\
\text { Exercise, } 10 \text { minutes } \\
\text { Post Exercise, } 7 \frac{1}{2} \text { minutes } \\
\text { Post Exercise, } 25 \text { minutes }\end{array}$ & $\begin{array}{r}3480 \\
-270 \\
-100 \\
-40 \\
+90\end{array}$ & $\begin{array}{l}-7.8 \\
-2.9 \\
-1.1 \\
+2.6\end{array}$ & $\begin{array}{r}6380 \\
-280 \\
-40 \\
+20 \\
+110\end{array}$ & $\begin{array}{l}-4.4 \\
-0.6 \\
+0.3 \\
+1.7\end{array}$ & $\begin{array}{l}2900 \\
-10 \\
+60 \\
+60 \\
+20\end{array}$ & $\begin{array}{l}-0.3 \\
+2.1 \\
+2.1 \\
+0.7\end{array}$ & $\begin{array}{l}45.4 \\
47.4 \\
46.7 \\
46.3 \\
45.0\end{array}$ & $\begin{array}{l}15.5 \\
16.4 \\
16.3 \\
16.0 \\
15.7\end{array}$ & $\begin{array}{l}5.72 \\
6.07 \\
6.39 \\
6.08\end{array}$ & $\begin{array}{l}196 \\
195 \\
216 \\
217\end{array}$ & \begin{tabular}{l|}
4.6 \\
4.7 \\
4.7 \\
4.7 \\
4.6
\end{tabular} & $\begin{array}{l}35 \\
93 \\
95 \\
40\end{array}$ & $\begin{array}{l}\text { Sitting, bioycle ergometer; } 330 \\
\text { kgm. per minute for } 10 \text { minutes. } \\
\text { Normal. }\end{array}$ \\
\hline $\begin{array}{c}\text { N. K. } \\
\text { February } 22,1938\end{array}$ & \begin{tabular}{|l|} 
Rest \\
Exercise, 5 minutes \\
Exercise, 10 minutes \\
Exercise, 16 minutes \\
Post Exercise, 12 minutes \\
Post Exercise, 27 minutes
\end{tabular} & $\begin{array}{r}3090 \\
-250 \\
-290 \\
-410 \\
-370 \\
-330\end{array}$ & $\begin{array}{r}-8.1 \\
-9.4 \\
-13.3 \\
-12.0 \\
-10.7\end{array}$ & $\begin{array}{r}5760 \\
-300 \\
-390 \\
-410\end{array}$ & $\begin{array}{l}-5.2 \\
-6.8 \\
-7.1\end{array}$ & $\begin{array}{l}2670 \\
-10 \\
+20 \\
-80\end{array}$ & $\begin{array}{l}-0.4 \\
+0.8 \\
+3.0\end{array}$ & $\begin{array}{l}46.4 \\
48.7 \\
50.1 \\
48.4\end{array}$ & \begin{tabular}{r|r|}
16.6 & \\
17.5 & 17.9 \\
17.9 & \\
16.9 &
\end{tabular} & $\begin{array}{l}6.9 \\
7.56 \\
7.6 \\
7.5\end{array}$ & $\begin{array}{l}213 \\
212 \\
204 \\
207\end{array}$ & \begin{tabular}{l|}
4.6 \\
4.8 \\
5.2 \\
4.7 \\
\end{tabular} & & $\begin{array}{l}\text { Sitting, bicycle ergometer; } 426 \\
\text { kgm. per minute for } 12 \text { minutes, } \\
\text { then } 830 \mathrm{kgm} \text {. per minute for } 4 \\
\text { minutes. } \\
\text { Normal. }\end{array}$ \\
\hline $\begin{array}{c}\text { N. K. } \\
\text { February } 20,1939\end{array}$ & \begin{tabular}{|l|} 
Rest \\
Exercise, 7 minutes \\
Exercise, 12 minutes \\
Exercise, 15 minutes \\
Post Exercise, 12 minutes \\
Post Exercise, 26 minutes
\end{tabular} & $\begin{array}{r}3280 \\
-230 \\
-130 \\
-200 \\
-30 \\
+30\end{array}$ & $\begin{array}{l}-7.0 \\
-4.0 \\
-6.1 \\
-0.9 \\
+0.9\end{array}$ & $\begin{array}{r}6200 \\
-270 \\
-80 \\
+50 \\
+140\end{array}$ & $\begin{array}{r}-4.4 \\
-1.3 \\
+0.8 \\
+2.3\end{array}$ & $\begin{array}{r}2920 \\
-40 \\
+50 \\
+80 \\
+110\end{array}$ & $\begin{array}{l}-1.4 \\
+1.7 \\
+2.7 \\
+3.8\end{array}$ & $\begin{array}{l}47.1 \\
48.1 \\
48.1 \\
47.5 \\
47.4\end{array}$ & $\begin{array}{l}15.1 \\
15.6\end{array}$ & \begin{tabular}{l|l|}
6.7 \\
7.02 \\
6.9
\end{tabular} & $\begin{array}{l}220 \\
221 \\
228\end{array}$ & & $\begin{array}{l}100 \\
158\end{array}$ & $\begin{array}{l}\text { Sitting, ohair ergometer; worked } \\
\text { with left arm for } 15 \text { minutes. } \\
\text { Normal. }\end{array}$ \\
\hline $\begin{array}{c}\text { V. D. } \\
\text { June 6, } 1939\end{array}$ & \begin{tabular}{|l|} 
Rest \\
Post Exercise, 1 minute \\
Post Exercise, 5 minutes \\
Post Exercise, 11 minutes \\
Post Exercise, 28 minutes
\end{tabular} & $\begin{array}{r}3210 \\
-670 \\
-500 \\
-490 \\
-400\end{array}$ & $\begin{array}{l}-20.9 \\
-15.6 \\
-15.3 \\
-12.5\end{array}$ & $\begin{array}{r}5800 \\
-520 \\
-300 \\
-240 \\
-230\end{array}$ & $\begin{array}{l}-9.0 \\
-5.2 \\
-4.1 \\
-4.0\end{array}$ & $\begin{array}{r}2590 \\
+150 \\
+200 \\
+250 \\
+170\end{array}$ & $\begin{array}{r}+5.8 \\
+7.7 \\
+9.7 \\
+6.6\end{array}$ & $\begin{array}{l}44.6 \\
51.9 \\
50.7 \\
51.0 \\
49.5\end{array}$ & $\begin{array}{l}14.9 \\
17.3 \\
16.5 \\
16.3\end{array}$ & $\begin{array}{l}6.32 \\
8.07 \\
7.34\end{array}$ & $\begin{array}{l}203 \\
205 \\
206\end{array}$ & $\begin{array}{l}4.1 \\
5.5 \\
5.2 \\
5.2\end{array}$ & $\begin{array}{r}64 \\
115 \\
30\end{array}$ & $\begin{array}{l}\text { Sitting, bicycle ergometer; worked } \\
\text { to exhaustion in } 51 \text { minutes. } \\
\text { Normal. }\end{array}$ \\
\hline
\end{tabular}


doubling the amount of work caused a greater diminution in the plasma volume. Two individuals who did exhaustive work showed a rather marked decrease in plasma volume.

The changes in cell volume were variable. During moderate exertion the cell volume was slightly diminished in all instances at the end of 5 or 6 minutes of work. Just before work was discontinued the volume of the cells showed a very slight increase in 2 cases (R. D. and C. M.).
When the exercise was prolonged, as in the case of $\mathrm{N}$. K., or exhaustive work was performed, the cell volume was increased in 2 out of 3 cases. The individual (V. D.) performing exhaustive exercise on the ergometer showed a moderate increase in cell volume.

Due chiefly to the loss of plasma from the blood stream, the degree of diminution in the blood volume was almost parallel to that of the plasma volume. During severe exercise, however, the

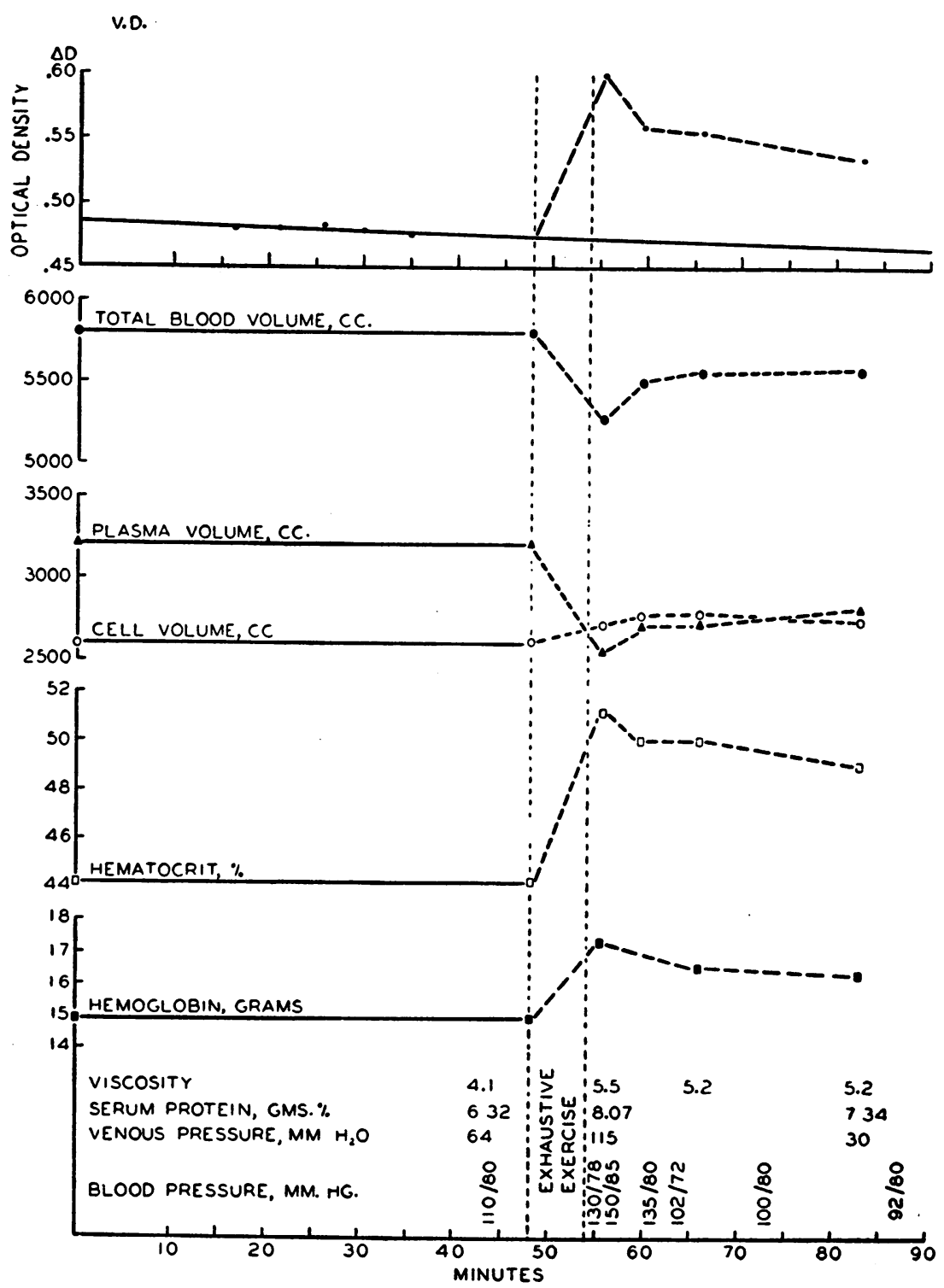

Fig. 2. The Changes in the Volume of the Blood and Other Related Circulatory measurements During and After Exhaustive Work by a NORMal INDIVIDUAL 
decrease in blood volume was not quite so great as that of the plasma volume because of the addition of red cells to the blood stream.

As a result of these alterations in the blood volume, the serum proteins, the hemoglobin, and the viscosity of the blood increased in proportion to the fluid loss from the blood stream. In 5 cases in which the arm-to-tongue time was measured during exercise, it was found to be decreased.

Following exercise the concentration of the dye in the serum gradually fell toward the prolongation of the disappearance slope, indicating dilution of the plasma. The plasma volume increased so that 25 minutes after exercise the volume was within +2.6 and -0.7 per cent of the initial value. Similar changes were noted in the blood volume. During the post-exercise period the alterations in the cell volume were again variable. Several cases showed a slight increase, others a slight diminution. Twenty-five minutes after exertion the hemoglobin and viscosity of the blood usually reached the pre-exercise level, while the serum protein was slightly higher than the control value.

When more severe exercise (N. K. and V. D.) was performed, the volumes of the plasma and blood were still diminished and, in 1 case (V. D.), the cell volume remained elevated 25 minutes after exercise (Figure 2). Likewise, the hemoglobin and viscosity of the blood and the serum proteins remained elevated, although the venous pressure was less than that observed during the control period.

Heart disease. The alterations in the blood volume due to exercise in patients with heart disease and those wtih poor vascular responses were very similar to those in the normal subjects (Table II). There was diminution in the plasma volume. The blood volume was not proportionally diminished because there was a slight increase in the cell volume. Here, too, the decrease in the plasma volume paralleled changes in the arterial and venous pressures. During the post-exercise period the plasma and blood volume gradually returned

TABLE II

Changes in the volume of the blood before, during, and after moderate exertion in abnormal subjects

\begin{tabular}{|c|c|c|c|c|c|c|c|c|c|c|c|c|c|c|}
\hline & & \multicolumn{6}{|c|}{ Change in volume } & $\begin{array}{l}\text { Hems- } \\
\text { toerit }\end{array}$ & $\begin{array}{l}\text { Hemo- } \\
\text { globin }\end{array}$ & $\begin{array}{c}\text { Berum } \\
\text { pro- } \\
\text { teins }\end{array}$ & $\begin{array}{l}\text { Total } \\
\text { cireu- } \\
\text { lating } \\
\text { pro- } \\
\text { teing }\end{array}$ & $\begin{array}{l}\text { Vis- } \\
\cos - \\
\text { ity }\end{array}$ & $\begin{array}{l}\text { Ve- } \\
\text { nous } \\
\text { pres- } \\
\text { sure }\end{array}$ & Remarks \\
\hline $\begin{array}{l}\text { J. C. } \\
\text { March 30, } 1939 \\
18 \text { years }\end{array}$ & $\begin{array}{l}\text { Rest } \\
\text { Post Exercise, } 2 \text { minutes } \\
\text { Post Erereise, } 5 \text { minutes } \\
\text { Post Exercise, } 15 \text { minutes } \\
\text { Post Erercie, } 25 \text { minutes }\end{array}$ & $\begin{array}{c}c c . \\
3250 \\
-410 \\
-330 \\
-230 \\
-310\end{array}$ & $\begin{array}{c}\text { per } \\
\text { cont } \\
\\
-12.6 \\
-10.2 \\
-7.1 \\
-0.5\end{array}$ & $\begin{array}{r}c c \\
5350 \\
-500 \\
-270 \\
-390\end{array}$ & $\begin{array}{c}\text { per } \\
\text { cent } \\
-9.4 \\
-5.1 \\
-7.3\end{array}$ & $\begin{array}{c}\infty \\
2100 \\
-80 \\
-40 \\
-80\end{array}$ & $\begin{array}{c}\text { per } \\
\text { cent } \\
\\
-4.3 \\
-1.9 \\
-3.8\end{array}$ & $\begin{array}{l}\text { per } \\
\text { cent } \\
39.3 \\
41.5 \\
40.5 \\
40.7\end{array}$ & grams & $\begin{array}{c}\text { orams } \\
\text { per } \\
\text { cont } \\
6.8 \\
6.9 \\
7.1\end{array}$ & $\begin{array}{c}\text { grame } \\
221 \\
202 \\
209\end{array}$ & & $\mathrm{~mm}_{\mathrm{H}} \mathrm{O}$ & $\begin{array}{l}\text { Sitting, exercise consigted of wallking } \\
\text { up and down stairs for } 7 \text { minutes. } \\
\text { Anorexia nervosa. }\end{array}$ \\
\hline $\begin{array}{l}\text { M. W. } \\
\text { March 15, } 1938 \\
\text { } 44 \text { years }\end{array}$ & \begin{tabular}{|l|} 
Rest \\
Exercise, 6 minutes \\
Exercise, 10 minutes \\
Post Exeroise, 6 minutes \\
Post Erervise, 25 minutes
\end{tabular} & $\begin{array}{r}2430 \\
-110 \\
-150 \\
-120 \\
-60 \\
\end{array}$ & $\begin{array}{l}-4.5 \\
-6.2 \\
-4.9 \\
-2.5\end{array}$ & $\begin{array}{r}4300 \\
-30 \\
-100 \\
-80 \\
-70\end{array}$ & $\begin{array}{r}-0.7 \\
-2.3 \\
-1.9 \\
-1.6 \\
\end{array}$ & $\begin{array}{r}1870 \\
+80 \\
+50 \\
+40 \\
-10\end{array}$ & $\begin{array}{l}+4.3 \\
+2.7 \\
+2.1 \\
-0.5 \\
\end{array}$ & $\begin{array}{l}43.5 \\
45.7 \\
45.7 \\
45.1 \\
44.0\end{array}$ & \begin{tabular}{r|}
14.3 \\
15.0 \\
14.6
\end{tabular} & $\begin{array}{l}6.8 \\
7.4 \\
7.3\end{array}$ & $\begin{array}{l}166 \\
169 \\
173\end{array}$ & $\begin{array}{l}4.1 \\
4.3 \\
4.1\end{array}$ & \begin{tabular}{r|}
87 \\
88 \\
107 \\
89 \\
\end{tabular} & $\begin{array}{l}\text { Sitting, bicycle ergometer; } 327 \mathrm{kgm} \text {. } \\
\text { per minute for } 10 \text { minutes. } \\
\text { Chronic alcoholism. }\end{array}$ \\
\hline $\begin{array}{c}\text { P. B. } \\
\text { March 26, } 1938 \\
10 \text { years }\end{array}$ & $\begin{array}{l}\text { Rest } \\
\text { Post Exercise, } 5 \text { minutes } \\
\text { Post Exrecise, } 26 \text { minutes }\end{array}$ & $\begin{array}{r}2620 \\
-150 \\
-5\end{array}$ & $\begin{array}{l}-5.7 \\
-0.2\end{array}$ & $\begin{array}{r}5090 \\
-170 \\
\end{array}$ & -3.3 & $\begin{array}{l}2470 \\
-20\end{array}$ & -0.8 & $\begin{array}{l}48.5 \\
49.8 \\
48.1\end{array}$ & $\begin{array}{l}17.1 \\
17.7 \\
17.2\end{array}$ & $\begin{array}{l}6.9 \\
7.3\end{array}$ & $\begin{array}{l}181 \\
180\end{array}$ & $\begin{array}{l}5.3 \\
5.3 \\
5.4\end{array}$ & & $\begin{array}{l}\text { Sitting, bicycle ergometer; } 396 \mathrm{kgm} . \\
\text { per minute for } 10 \text { minutes. } \\
\text { Rheumstic heart disease, mitral } \\
\text { stenosis, and insufficieney. }\end{array}$ \\
\hline $\begin{array}{c}\text { G. H. } \\
\text { Pebruary } 25,1938 \\
40 \text { years }\end{array}$ & \begin{tabular}{|l|} 
Rest \\
Exereise, 6 minutes \\
Exereise, 9 minutes \\
Post Ererejo, 71 minutes \\
Post Erereise, 25 minutes
\end{tabular} & $\begin{array}{r}3220 \\
-260 \\
-340 \\
-90 \\
-50\end{array}$ & $\begin{array}{r}-8.1 \\
-10.5 \\
-2.8 \\
-1.6\end{array}$ & $\begin{array}{r}5740 \\
-20 \\
-180 \\
-50\end{array}$ & $\begin{array}{r}-0.3 \\
-3.1 \\
-0.9\end{array}$ & $\begin{array}{r}2520 \\
+240 \\
+160 \\
0\end{array}$ & $\begin{array}{c}+9.5 \\
+6.3 \\
0\end{array}$ & $\begin{array}{l}43.9 \\
48.2 \\
48.1 \\
44.3\end{array}$ & $\begin{array}{l}14.7 \\
16.0 \\
15.0\end{array}$ & $\begin{array}{l}5.7 \\
6.4 \\
5.9\end{array}$ & $\begin{array}{l}184 \\
184 \\
187\end{array}$ & $\begin{array}{l}4.2 \\
4.9 \\
4.1\end{array}$ & $\begin{array}{r}68 \\
105 \\
85 \\
63\end{array}$ & $\begin{array}{l}\text { Sitting, bicycle ergometer; } 334 \mathrm{kgm} \text {. } \\
\text { per minute for } 10 \text { minutes. } \\
\text { Rheumatic heart disease, mitral } \\
\text { stenosis, mitral insufficiency, } \\
\text { aortio insufficienoy, suricular } \\
\text { fibrillation. }\end{array}$ \\
\hline $\begin{array}{l}\text { H. C. } \\
\text { March 4, } 1938 \\
16 \text { years }\end{array}$ & $\begin{array}{l}\text { Rest } \\
\text { Exercise, } 6 \text { minutes } \\
\text { Exeroiee, } 10 \text { minutes } \\
\text { Post Exercise, } 71 \text { minutes } \\
\text { Post Exercise, } 25 \text { minutes }\end{array}$ & $\begin{array}{r}2960 \\
-230 \\
-220 \\
-110 \\
-90\end{array}$ & $\begin{array}{l}-7.8 \\
-7.4 \\
-3.7 \\
-3.0\end{array}$ & $\begin{array}{r}5410 \\
-210 \\
-190 \\
-100 \\
-100\end{array}$ & $\begin{array}{l}-3.9 \\
-3.5 \\
-1.9 \\
-1.9\end{array}$ & $\begin{array}{l}2450 \\
+20 \\
+10 \\
+10 \\
-10\end{array}$ & $\begin{array}{l}+0.8 \\
+0.4 \\
+0.4 \\
-0.4\end{array}$ & $\begin{array}{l}45.3 \\
47.5 \\
47.5 \\
45.8 \\
45.3\end{array}$ & $\begin{array}{l}16.0 \\
17.2 \\
17.2 \\
16.8 \\
16.4\end{array}$ & $\begin{array}{l}6.3 \\
6.79 \\
6.89\end{array}$ & $\begin{array}{l}187 \\
186 \\
198\end{array}$ & $\begin{array}{l}4.3 \\
4.6 \\
4.7 \\
4.4 \\
4.5\end{array}$ & $\begin{array}{r}97 \\
183\end{array}$ & $\begin{array}{l}\text { Sitting, bicycle ergometer; } 348 \mathrm{kgm} \text {. } \\
\text { per minute for } 10 \text { minutes. } \\
\text { Rheumatic beart disease, mitral } \\
\text { stenosis, mitral insufficiency, and } \\
\text { aortic inouffieiency. }\end{array}$ \\
\hline$\underset{\substack{\text { April } 27,1939 \\
\text { 55 years }}}{\text { R. }}$ & $\begin{array}{l}\text { Rest } \\
\text { Post Erereice, } 1 \text { minute } \\
\text { Post Brencise, } 14 \text { minutes } \\
\text { Post Brenvioe, } 27 \text { minutes }\end{array}$ & $\begin{array}{r}3430 \\
-280 \\
-50 \\
+30\end{array}$ & $\begin{array}{l}-8.2 \\
-1.5 \\
+0.9\end{array}$ & $\begin{array}{r}6210 \\
-150 \\
+120\end{array}$ & $\begin{array}{r}-2.4 \\
+1.9\end{array}$ & $\begin{array}{r}2780 \\
+130 \\
+80\end{array}$ & $\begin{array}{l}+4.7 \\
+3.2\end{array}$ & $\begin{array}{l}44.8 \\
48.0 \\
45.3\end{array}$ & $\begin{array}{l}15.5 \\
16.8 \\
16.3\end{array}$ & $\begin{array}{l}6.3 \\
7.1 \\
6.4\end{array}$ & $\begin{array}{l}216 \\
224 \\
221\end{array}$ & & & $\begin{array}{l}\text { Sitting, exercise consisted of walking } \\
\text { up and down stairs for } 81 \text { minutes } \\
\text { Hypertengive heart disease Lungs } \\
\text { clear. Slight pretibial edema. }\end{array}$ \\
\hline
\end{tabular}


toward the resting values. Cases J. C. and M. W. showed a more marked failure to regain the necessary plasma volume level than did most of the cardiac subjects. Parallel changes were taking place in regard to serum proteins, hemoglobin and the viscosity of the blood.

If, on the assumption that the plasma proteins do not enter or leave the circulating blood during these procedures, the changes in plasma volume during the experimental period can be calculated from the values of the serum proteins, the results thus obtained are in fairly good accord with those obtained directly by the dye method, except in 2 cases (C. M. and J. C.). No consistent differences between the two methods were noted which could be interpreted to indicate that protein left and entered the blood stream during or after exercise (Table III).

TABLE III

Comparison of the values observed for the plasma protein with those calculated from the changes in total plasma volume

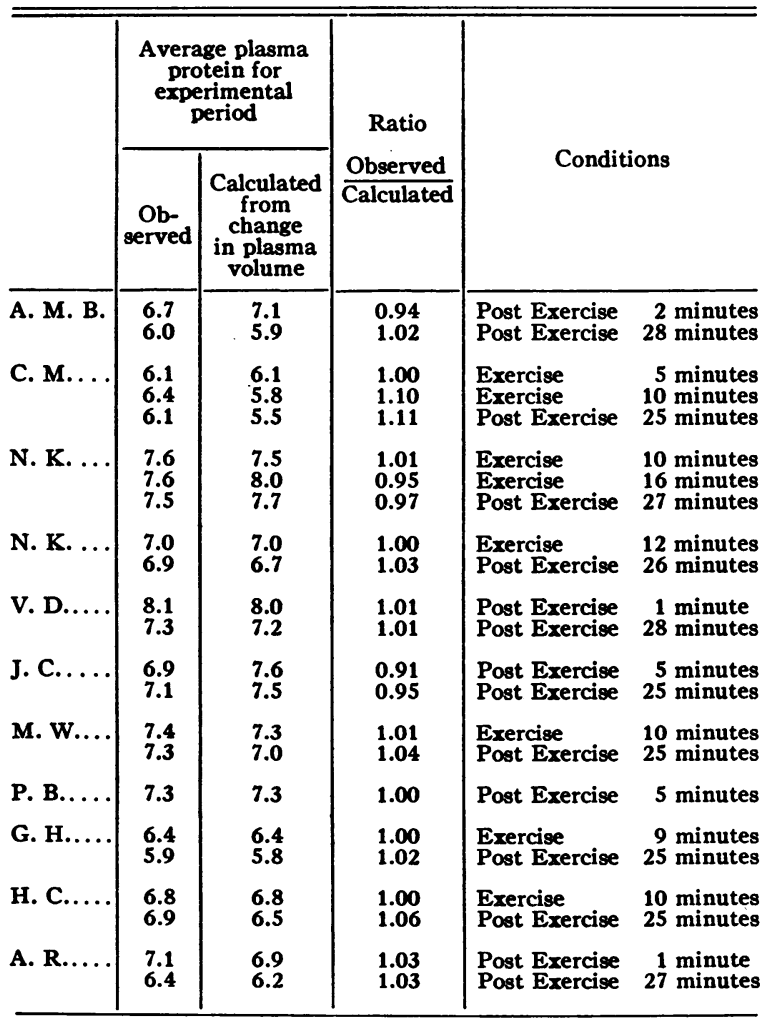

\section{DISCUSSION}

These results in general are at variance with those reported in the literature, except in a few in- stances where indirect studies of the blood volume were made $(4,5)$. The discrepancies in the results obtained by us and those of previous investigators arise, we believe, from errors in the earlier techniques and procedures employed. The criticisms pointed out by Gregersen, Gibson, and Stead and others $(19,14)$ are particularly true when plasma volumes are estimated within short intervals and at times when the circulating blood volume is changing rapidly. In contrast to earlier methods, the one used in these experiments aptly lends itself to a study of changes occurring during experimental procedures. In the first place, ample time ( 15 to 20 minutes) is given for the dye to be completely mixed with the plasma before variations in its volume are brought about; secondly, it is not necessary to inject more dye at the time when the plasma volume is rapidly changing ; and, finally, alterations produced can be followed both during and after the exercise period.

In contrast to the conflicting results reported in the literature, the observations presented above show a consistency in the direction and extent of changes of the plasma and blood volumes during and following short periods of moderate exertion in both normal subjects and individuals with heart disease. During muscular exertion the plasma volume diminishes. The decrease is proportional to the severity of the exercise. The decrease in the plasma volume is due to the sudden shift of fluid from the vascular system to the interstitial fluid and the active muscles. This transfer of fluid is attributed to the rise in systemic pressure and the hyperemia of the muscle which results in a rise of the local and capillary pressure. This in turn leads to a disturbance in the filtration-absorption equilibrium of fluids in the capillaries (20). As the blood passes through the active muscles, more fluid is forced out into the tissues than can be immediately absorbed. Some of the fluid presumably goes into the muscle cells $(4,20,21)$. After cessation of exercise, the process reverses.

Consistent findings were also observed in the cell volume as a result of exercise. No new cells were added to the circulation during moderate exertion in normal individuals. Therefore, it is evident that the increase in the red blood cells and hemoglobin during work is due to a concentration of the blood. Furthermore, these observations support the view that the increased volume of the 
blood supplied to the active muscles is brought about mainly by a redistribution rather than by an increase in the volume of the circulating blood. When, however, the amount of work is greater, depending on the capacity of the subject-whether normal or with diminished cardiac reserve-then not only is the plasma volume diminished but also the cell volume is increased. But even under these circumstances the diminution in the plasma volume is always more marked than the increase in the cell volume (Case V. D.).

There is some evidence to indicate that the moderate increase in cell volume during exhaustive exercise may be due to the addition of red cells from the blood reservoirs to the circulation. In animals there is ample proof that exercise causes the spleen to contract and discharge cells into the blood stream (3). It has been shown that this contraction is mediated through the nervous system and that either lack of oxygen or an increased secretion of adrenin may act as the stimulus. However, in man, the depot function of the spleen and other organs may be relatively unimportant, since during exercise, when presumably the reserves are called out, the circulating cell volume is not greatly increased even when the exercise is severe. From the experimental data available it appears that the stimulus for the contraction of the blood depots during severe exercise in man is due to the sympathico-adrenal mechanism rather than lack of oxygen (22). It has been shown that the subcutaneous administration of epinephrine is followed by a prompt rise in the cell volume (23), while lack of oxygen requires a relatively long time (several hours to days) for it to become effective in increasing the cell volume (24). Exhaustive locomotor activity by normal subjects and moderate activity by individuals with poor vascular control are probably associated with emotional excitement, an excellent stimulus for increased secretion of adrenin (22).

Finally, it must be pointed out that the cell volume is not measured directly by this method. The calculations of the blood and cell volumes are based on the ratio of cells to plasma as drawn from the antecubital vein. There is sufficient evidence to indicate that the red cells and the plasma are not thoroughly and uniformly mixed within the vascular system (25). Until more data are available concerning the alteration that may take place in the ratio of cells to plasma in various parts of the vascular system during exercise, one does not know how much reliance to place upon the above changes in cell volume.

\section{SUMMARY AND CONCLUSIONS}

Determinations of the volume of the blood were made at rest and variations in this volume were followed during and after varying grades of exercise in normal subjects and in individuals suffering with cardiac disease. Additional observations included measurements of the blood hemoglobin and viscosity, serum proteins, and venous pressure. The results of this investigation lead to the following conclusions:

1. In normal individuals during moderate exertion there is a prompt and definite decrease in the plasma volume, accompanied by a corresponding decrease in the blood volume, while the changes in the cell volume are variable though slight. These changes are associated with an increase in the blood hemoglobin and viscosity, the serum proteins and the venous and arterial pressures. Following exercise the plasma volume gradually increases and 25 minutes after exercise the plasma volume, blood hemoglobin, and serum proteins reach the pre-exercise values.

2. During exhaustive exercise in normal subjects, there is a further decrease in plasma volume accompanied by a moderate increase in the cell volume. Twenty-five minutes after the cessation of exercise the plasma volume is still diminished and the blood hemoglobin and serum proteins are increased.

3. In patients with compensated heart disease, the changes in the blood volume during and following exercise are similar to those of normal subjects.

4. The increase in red blood cells and hemoglobin concentration resulting from exercise is brought about mainly by passage of protein-poor fluid from the vascular system into the interstitial spaces. It is only during severe or exhaustive exercise that new cells are added to the circulating blood.

\section{BIBLIOGRAPHY}

1. Hawk, P. B., On the morphological changes in the blood after muscular exercise. Am. J. Physiol., 1904, 10, 384. 
2. Schneider, E. C., and Havens, L. C., Changes in the blood after muscular activity and during training. Am. J. Physiol., 1915, 36, 239.

3. Barcroft, J., and Stephens, J. G., Observations upon the size of the spleen. J. Physiol., 1927, 64, 1.

4. Scott, F. H., Herrmann, E. T., and Snell, A. M., Factors influencing the interchange of fluid between blood and tissue spaces. II. Muscular activity. Am. J. Physiol., 1917, 44, 313.

5. Dill, D. B., Talbott, J. H., and Edwards, H. T., Studies in muscular activity. VI. Response of several individuals to a fixed task. J. Physiol., 1930, 69, 267.

6. Lee, F. W., Whipple, G. H., and Carrier, E. B., Simultaneous determinations of plasma and hemoglobin volumes; influence of fluids by mouth and vigorous exercise. Am. J. Physiol., 1922, 61, 149.

7. Broun, G. O., Blood destruction during exercise; I. Blood changes occurring in the course of a single day of exercise. J. Exper. Med., 1922, 36, 481.

8. Chang, H. C., and Harrop, G. A., Jr., The determination of the circulating blood volume with carbon monoxide. J. Clin. Invest., 1928, 5, 393.

9. Ewig, W., and Hinsberg, K., Krieslaufstudien II. Neue Methode zur Bestimmung des Herzminutenvolumens. Ztschr. f. klin. Med., 1930-31, 115, 693.

10. Wollheim, E., Die Zirkulierende Blutmenge und ihre Bedentung fur Kompensation und Dekompensation des Krieslaufs. Ztschr. f. klin. Med., 1931, 116, 269.

11. Levin, E., Influencia del ejercicio muscular sobre el volumen de sangre circulante en estados normales y pathologicos. Rev. Soc. Argent. de Biol., 1935, 11, 83.

12. Lozoya, S. J., El volumen de plasma y de sangre circulantes en las altitudes y sus variaciones con el tiempo y con el ejercicio muscular. Arch. Latino. Am. Cardiol. y Hemat., 1936, 6, 241.

13. Gibson, J. G., 2nd, and Branch, C. D., Blood volume changes during surgical procedures. Surg. Gynec. and Obst., 1937, 65, 741.
14. Gibson, J. G., 2nd, and Evans, W. A., Jr., Clinical studies on the blood volume. I. Clinical application of a method employing the azo dye "Evans blue" and spectrophotometer. J. Clin. Invest., 1937, 16, 301.

15. Van Slyke, D. D., and Neill, J. M., The determination of gases in blood and other solutions by vacuum extraction and manometric measurement. J. Biol. Chem., 1924, 61, 523.

16. Howe, P. E., The determination of proteins in blood; a micro-method. J. Biol. Chem., 1921, 49, 109.

17. Tarr, L., Oppenheimer, B. S., and Sager, R. V., The circulation time in various clinical conditions determined by the use of sodium dehydrocholate. Am. Heart J., 1933, 8, 766.

18. Griffith, G. C., Chamberlain, C. T., and Kitchell, J. R., A simplified apparatus for direct venous pressure determination modified from Mortiz and v. Tabora. Am. J. M. Sc., 1934, 187, 371.

19. Gregersen, M. I., Gibson, J. J., and Stead, E. A., Plasma volume determination with dyes; errors in colorimetry; use of the blue dye T-1824. Am. J. Physiol. (Proc.), 1935, 113, 54.

20. Barcroft, J., and Kato, T., Effects of functional activity in striated muscle and the submaxillary gland. Phil. Trans. Royal Soc., B. 1915, 207, 149.

21. Fenn, W. O., and Cobb, D. M., Electrolyte changes in muscle during activity. Am. J. Physiol., 1936, 115, 345.

22. Cannon, W. B., The Wisdom of the Body. W. W. Norton and Co., New York, 1932.

23. Kaltreider, N. L., Meneely, G. R., and Allen, J., The effect of adrenalin on the blood volume. (In preparation.)

24. Smith, H. P., Belt, A. E., Arnold, H. R., and Carrier, E. B., Blood volume changes at high altitudes. Am. J. Physiol., 1924-25, 71, 395.

25. Smith, H. P., Arnold, H. R., and Whipple, G. H., Blood volume studies; VII. Comparative values of Welcker, carbon monoxide, and dye methods for blood volume determinations; accurate estimation of absolute blood volume. Am. J. Physiol., 1921, 56, 336. 